\title{
Spectral features selection and classification of oil palm leaves infected by Basal stem rot (BSR) disease using dielectric spectroscopy
}

\begin{abstract}
Basal stem rot (BSR) is a prominent plant disease caused by Ganoderma boninense fungus, which infects oil palm plantations leading to large economic losses in palm oil production. There is need for novel disease detection techniques that can be used to reduce the oil palm losses due to BSR. Thus, this paper investigated the feasibility of utilizing electrical properties such as impedance, capacitance, dielectric constant, and dissipation factor in early detection of BSR disease in oil palm tree. Leaf samples from different oil palm trees (healthy, mild, moderate, and severely-infected) were collected and measured using a solid test fixture (16451B, Keysight Technologies, Japan) connected to an impedance analyzer (4294A, Agilent Technologies, Japan) at a frequency range of $100 \mathrm{kHz}-30 \mathrm{MHz}$ with 300 spectral interval. Genetic algorithm (GA), random forest (RF), and support vector machine-feature selection (SVM-FS) were used to analyze the electrical properties of the dataset and the most significant frequencies were selected. Following the selection of significant frequencies, the features were evaluated using two classifiers, support vector machine (SVM) and artificial neural networks (ANN) to determine the overall and individual class classification accuracies. The selection model comparative feature analysis demonstrated that the best statistical indicators with overall accuracy $(88.64 \%)$, kappa (0.8480) and low mean absolute error $(0.1652)$ were obtained using significant frequencies produced by SVM-FS model. The results indicated that the SVM classifier shows better performance as compared to ANN classifier. The results also showed that the classes, features selection models, and the electrical properties were found to be significantly different $(\mathrm{p}<.1)$. The impedance values were highly classified by Ganoderma disease at different levels of severity with overall accuracies of more than $80 \%$. Impedance can be considered as the best electrical properties that can be used to estimate the severity of BSR disease in oil palm using spectroscopy technique. As such, this study demonstrates the potentials of utilizing electrical properties for detection of Ganoderma diseases in oil palm.
\end{abstract}

Keyword: Basal stem rot disease; Dielectric properties; Oil palm; Support vector machine; Artificial neural network 